\title{
From Azidoproline to Functionalizable Collagen
}

\author{
Christiane Siebler, Roman S. Erdmann, and Helma Wennemers*
}

\begin{abstract}
The article summarizes our research devoted to the development of functionalizable collagen using azidoproline-containing model peptides. 'Click chemistry' or Staudinger reduction followed by acylation provided facile access to a range of differently functionalized collagen model peptides bearing e.g. carbohydrates. The research provided not only insight into the factors that are responsible for the high conformational stability of collagen but also a guide in which positions functional moieties are tolerated without or by deliberately disturbing the supramolecular assembly of collagen. The presented research will be useful for the development of collagenbased materials.
\end{abstract}

Keywords: CD spectroscopy · Click chemistry · Collagen · Peptides · Proline

\section{Introduction}

Collagen, the most abundant protein in mammals, is the most prevalent constituent of the extracellular matrix and provides for structural stability in disparate tissues and organs. ${ }^{[1]}$ Collagens also play crucial roles in the modulation of cellular activity, e.g. by binding and releasing cellular mediators such as cytokines and growth factors. ${ }^{[1]}$ Understanding the molecular factors that provide for the stability of collagen is therefore important. In addition, synthetic collagen-based materials are promising tools for the development of biocompatible materials that might be valuable for applications in e.g. tissue engineering, wound healing and as delivery systems. ${ }^{[2]}$ Whereas biotechnological approaches towards such materials rely on natural building blocks, organic synthesis is an attractive alternative since it offers the possibility of functionalizing collagen with essentially any desired building block.

Collagen consists of single strands of repeating Xaa-Yaa-Gly units that adopt polyproline II (PPII) like secondary structures and wrap around each other to form triple helices that further assemble into macroscopic fibers and bundles. ${ }^{[1]}$ Proline (Pro) is most abundant in the Xaa position and (4R)-hydroxyproline (Hyp) in the Yaa

\footnotetext{
${ }^{*}$ Correspondence: Prof. H. Wennemers Laboratorium für Organische Chemie D-CHAB, ETH Zürich

Wolfgang-Pauli-Strasse $10, \mathrm{CH}-8093$ Zürich Tel.: +41-44-633-3777
}

E-mail: Helma.Wennemers@org.chem.ethz.ch position. The cyclic nature of these imino acids enforces $\Phi$ torsion angles around $-75^{\circ}$ and favors $\Psi$ angles of $\sim 145^{\circ}$ that are ideal for PPII helices (Fig. 1). ${ }^{[1,3]}$

Crystal structures showed that the Pro residues in the Xaa positions adopt mainly C4-endo ring puckers whereas the Hyp residues in the Yaa positions are C4-exo ring-puckered. ${ }^{[4]}$ Glycine (Gly) is critical in every third position since the tight packing of the triple helices does not allow for a bulkier amino acid in their center. Within the triple helices the single strands are held together by interstrand hydrogen bonds between the $\mathrm{C}=\mathrm{O}$ of the Xaa position and the $\mathrm{NH}$ of Gly of the neighboring strand. ${ }^{[1]}$

Our group has a long standing interest in proline containing peptides (Fig. 2). ${ }^{[5-12]}$ We have, for example, established tripeptides of the type H-Pro-Pro-Aaa (Aaa = any amino acid) as highly stereoselective catalysts for aldol and conjugate addition reactions. ${ }^{[5,6]}$

In addition, we developed prolinebased diketopiperazine receptors for the

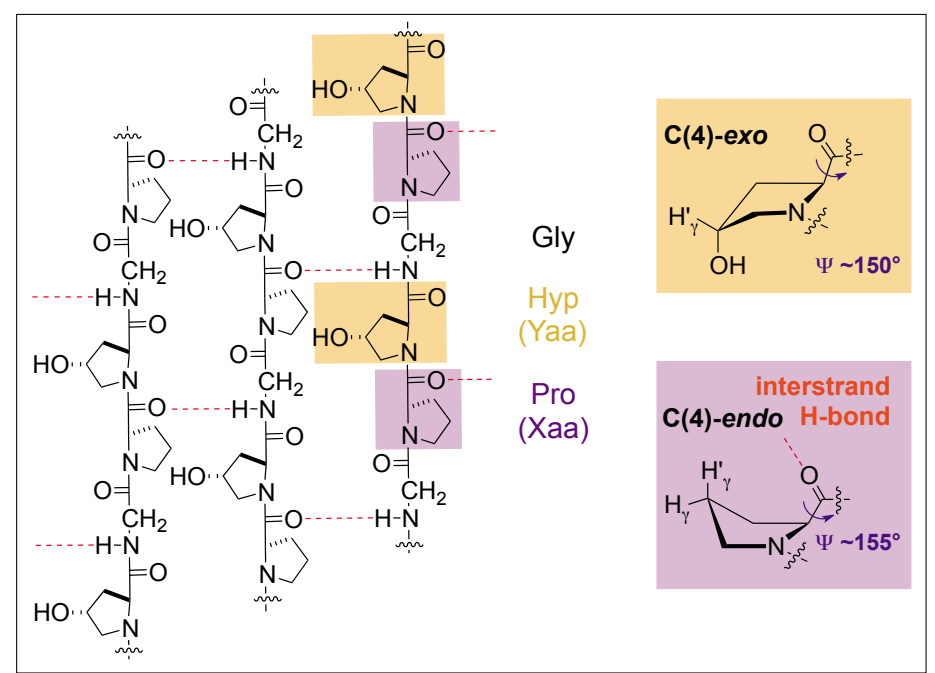

selective binding of short peptides ${ }^{[7]}$ and azidoproline (Azp) containing oligoprolines as conformationally well-defined molecular scaffolds that can be easily functionalized in defined distances. ${ }^{[8]}$ These functionalizable scaffolds are valuable for the controlled formation of silver nanoparticles and biological applications such as the development of cell-penetrating peptides, tumor targeting and selective RNA binding. ${ }^{[9]}$ Our studies on functionalized oligoprolines also provided insight into the factors that stabilize the PPII secondary structure and provided a guide for tuning the conformational properties of PPII helices. ${ }^{[8,10]}$ The facile functionalizability of the Azp residues within the oligoprolines combined with the unique conformational properties of Azp ${ }^{[11]}$ and derivatives thereof ${ }^{[12]}$ led us envision that Azp residues might also be valuable for the development of functionalized collagen. Within this article we summarize our contributions to the development of functionalizable collagen and the insights into the molecular factors
Fig. 1. General structure of the collagen triple helix (left) and preferred ring puckers of the Pro and Hyp residues in the Xaa and Yaa positions, respectively (right). 


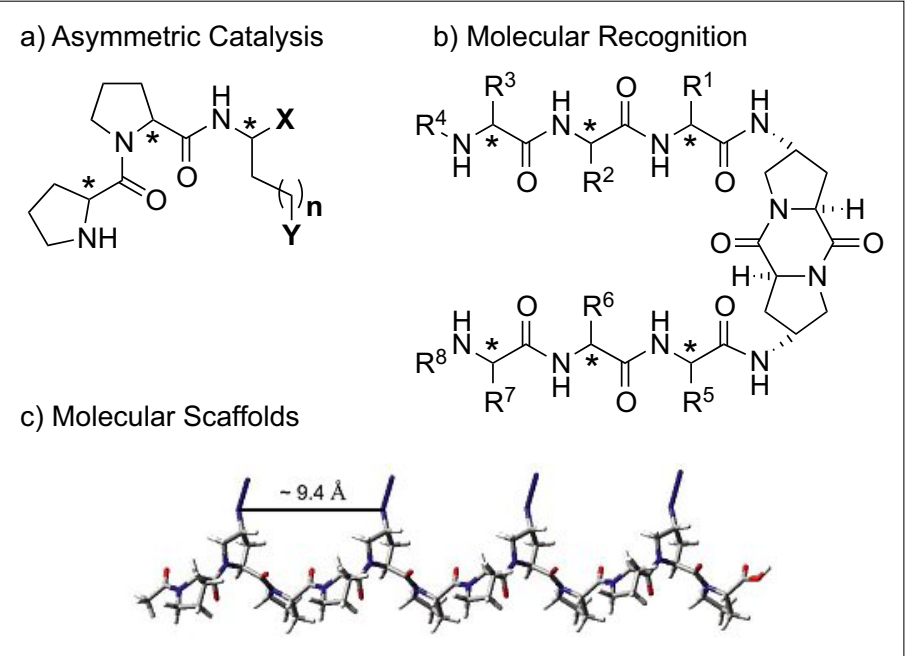

Fig. 2. Prolylpeptides in (a) asymmetric catalysis, (b) molecular recognition and (c) as functionalizable molecular scaffolds.

determining the conformational stability of collagen that were gained from these studies.

\section{Design and Synthesis of Functionalizable Collagen Model Peptides}

Since the environment of the Xaa and the Yaa positions within the collagen triple helix differ significantly from each other, incorporation of functionalized Azp residues in either of these positions will have distinctly different effects on the conformational stability of the collagen triple helix. In addition, the absolute configuration as well as the functional moiety at $\mathrm{C}(4)$ of proline residues are known to influence the ring pucker and the trans:cis ratio of XaaPro bonds and will therefore also affect the properties of the collagen triple helix. ${ }^{[11,13]}$ To probe the effect of functional groups on the collagen triple helix we hence prepared functionalizable CMPs bearing (4R)Azp or $(4 S)$ Azp residues either in the Xaa or the Yaa position of the middle repeat unit within 21-mers. ${ }^{[14,15]}$ Such CMPs allow for monitoring the relative effect of a single residue on the stability of the collagen triple helix. These CMPs were then derivatized by either $\mathrm{Cu}(\mathrm{I})$-catalyzed Huisgen's 1,3-dipolar cycloadditions ('click chemistry') or Staudinger reduction followed by acylation (Fig. 3). [15-19]

The synthetic routes are exemplified for the CMPs YR-3 and YR-7 that are functionalized via amide and triazolyl linkages, respectively, in the Yaa position (Scheme 1).

The syntheses of both types of CMPs, with amide and triazolyl linkers, were straightforward and allowed for functionalization with any desired moiety (e.g. bulky tert-butyl groups or monosaccha-

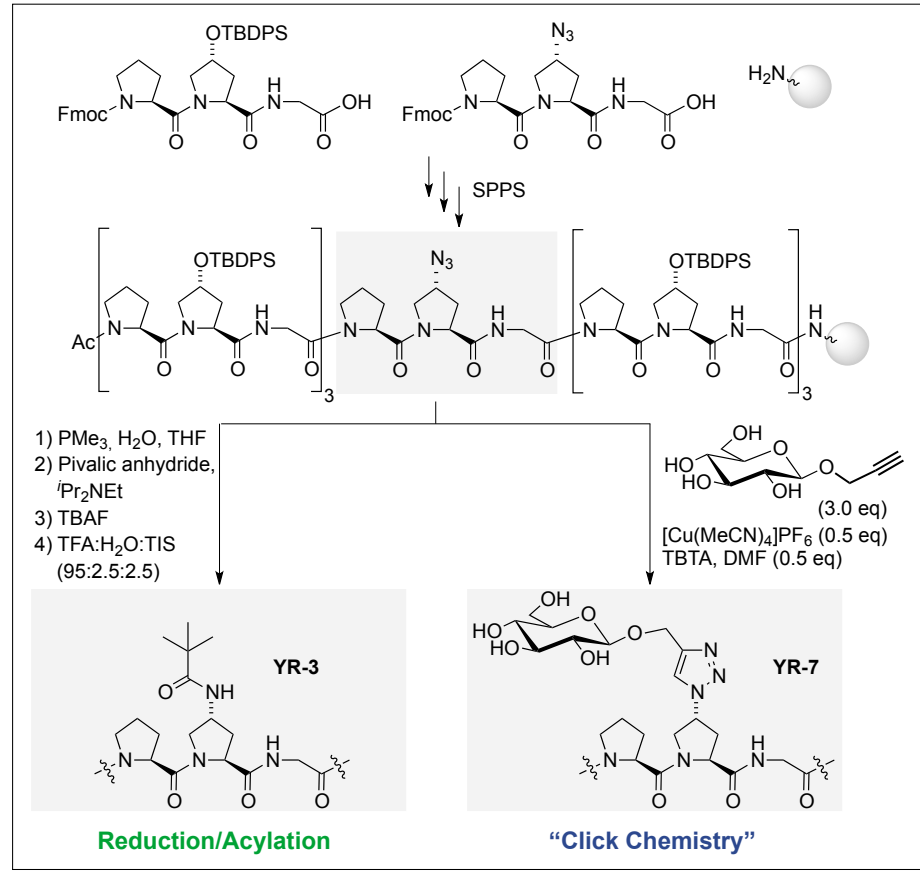

Scheme 1. Synthetic routes to functionalized collagen model peptides exemplified for YR-3 and YR-7. rides) to access the CMPs depicted in Fig. methylesters (Ac-Aaa-OMe) are valuable 3. [15-19]

\section{Conformational Properties of (4R)- and (4S)Azidoproline and their Derivatives}

To understand the effect of a modified proline residue on the stability of the collagen triple helix, the acetylated

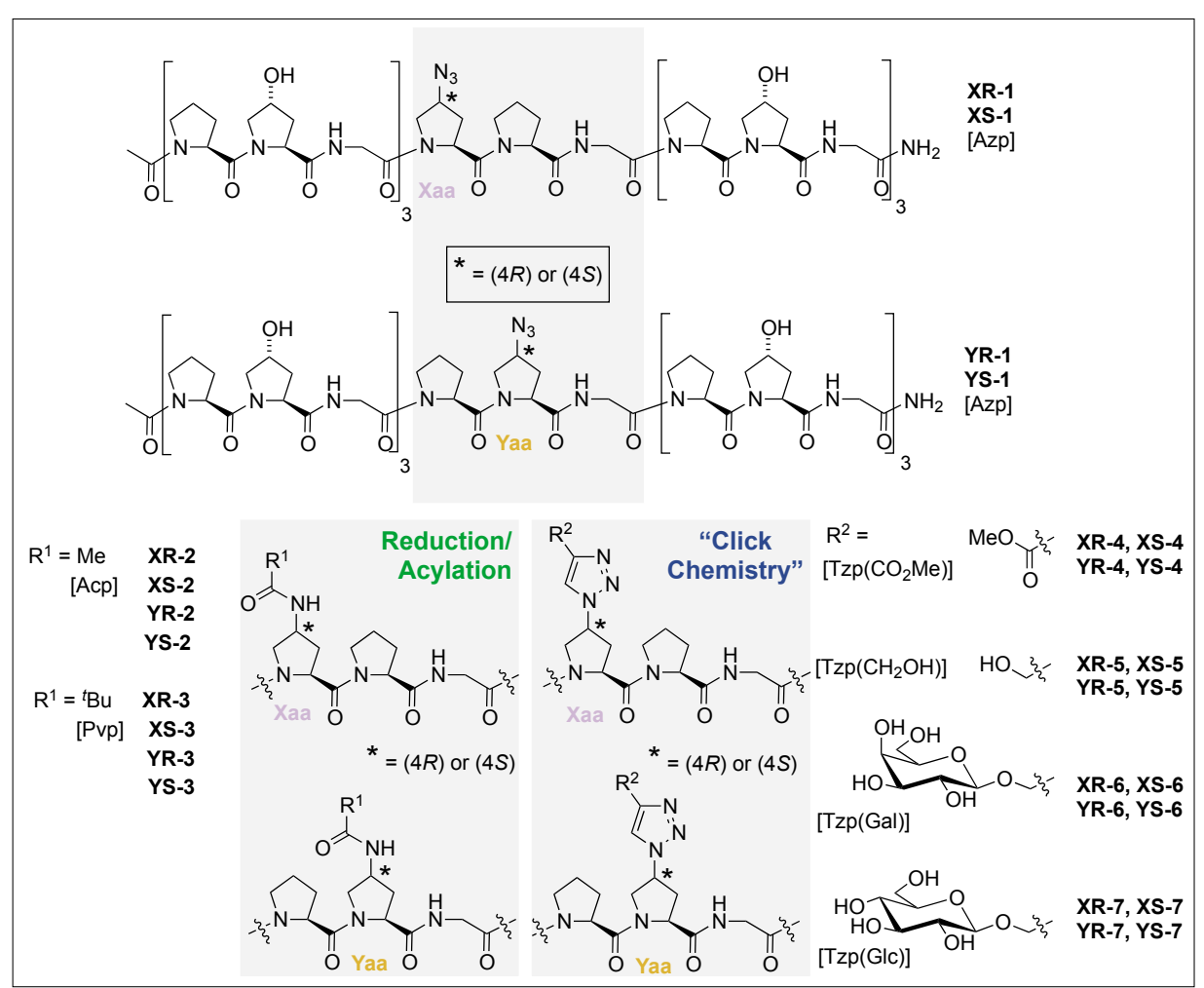

Fig. 3. Functionalized collagen model peptides. model compounds. ${ }^{[13]}$ We therefore carefully examined the conformational properties of Ac- $(4 S)$ Azp-OMe and Ac- $(4 R)$ Azp-OMe ${ }^{[11]}$ as well as those of amidated and triazolylated derivatives thereof (Fig. 4). ${ }^{[12,16,17]}$ A combination of NMR and IR spectroscopic analyses as well as ab initio calculations revealed that the ring puckering and the trans:cis amide bond ratio depend significantly on the absolute configu- 


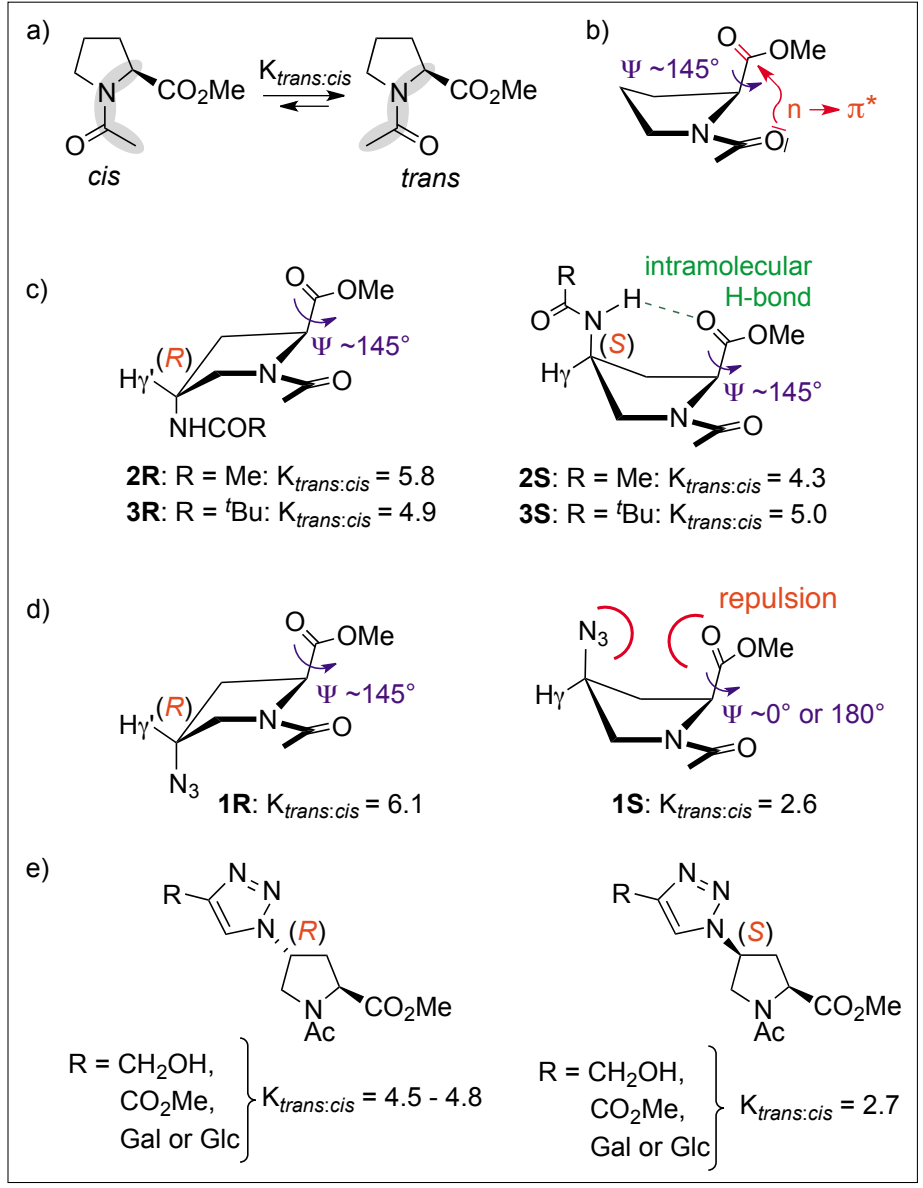

ration and the functional group attached at $\mathrm{C}(4)$. Both the ring puckering and the trans:cis amide bond ratio are controlled by a combination of steric effects, stereoelectronic gauche effects between the substituents at $\mathrm{C}(4)$ and the $\mathrm{N}$-acetyl group as well as repulsive or attractive interactions between the substituent at $\mathrm{C}(4)$ and the methylester. ${ }^{[11-13]}$

Electron-withdrawing groups at $\mathrm{C}(4)$ such as amide and in particular azido moieties exert gauche effects and lead to a preference for $\mathrm{C}(4)$-endo puckering in case of (4S)-configured and $\mathrm{C}(4)$-exo puckering in case of $(4 R)$-configured proline derivatives (Fig. 4c,d).[11,12] The effects observed with the azidoproline derivatives (1R and 1S) are comparable to those of the respective hydroxy- and fluoroproline derivatives ${ }^{[13 a-d]}$ underlining that the azido-gauche effect is comparable to that of hydroxy and fluoro groups. The exo-ring pucker within $(4 R)$ configured Azp (1R) and acylated aminoproline derivatives ( $\mathbf{2}$ and $\mathbf{3 R}$ ) allows for an $n \rightarrow \pi^{*}$ interaction ${ }^{[20]}$ between the acetyl group and the carbonyl group of the methylester within the trans conformer (Fig. 4b). ${ }^{[11,12]}$ As a result, the trans conformer is favored in these derivatives over the cis conformer by a factor of 5-6 in aqueous environments (Fig. 4c,d, left). ${ }^{[11,12]}$ The $\mathrm{n} \rightarrow \pi^{*}$ interaction that favors and stabilizes the trans conformer requires a BürgiDunitz trajectory between the oxygen of
Fig. 4. Conformational properties of model compounds Ac-AaaOMe with Aaa $=$ Acp or Pvp (c), Azp (d) and Tzp (e).
$\mathrm{C}(4)$-exo or $\mathrm{C}(4)$-endo ring puckering nor the differences in the trans:cis conformer ratios between $(4 R)$ - and $(4 S)$-configured derivatives are as pronounced as in case of the azido and amidoproline derivatives (Fig. 4e).[16,17]

\section{Thermal Stabilities of Functionalized Collagen Triple Helices}

CD-spectroscopic studies are a convenient method to monitor the formation of collagen triple helices that have a characteristic maximum at $225 \mathrm{~nm}$. ${ }^{1]}$ Such analyses showed that all of the functionalized CMPs depicted in Fig. 3 form triple helices. ${ }^{[15-19]}$ Thermal denaturation studies revealed that the relative stabilities of these collagen triple helices differ as expected significantly. The midpoints of thermal transitions $\left(\mathrm{T}_{\mathrm{m}}\right)$ within the sigmoidal curves recorded for each of the triple helices varied between $26-44{ }^{\circ} \mathrm{C}$ and the free energy values $(\Delta \mathrm{G})$ that were derived from additional denaturation experiments in which also the refolding process upon cooling the samples was monitored[21] varied between -7 and -12 kcal mol ${ }^{-1}$ (Fig. 5 and Table 1).[15-19]

Reassuringly, the relative differences between the $\Delta \mathrm{G}$ values and the $\mathrm{T}_{\mathrm{m}}$ values were in very good agreement. This observation underlined the value of the qualitative $T_{m}$ values that are significantly easier to obtain than the quantitative $\Delta \mathrm{G}$ values. This holds true as long as the same heating rates were used for the determination of the $\mathrm{T}$ values. ${ }^{[12,19]}$ For all of these studies CMP $\mathbf{8}$ with the naturally most abundant $(4 R) \mathrm{Hyp}$ residue as well as CMP 9 with Pro in the Yaa position were used as references (8: Ac[ProHypGly] ${ }_{7} \mathrm{NH}_{2}$, 9: Ac[ProHypGly] ProProGly-[ProHypGly] ${ }_{3} \mathrm{NH}_{2}$, Table 1 , entries 25 and 26). The triple helices derived from these CMPs have $T_{m}$ values of $43{ }^{\circ} \mathrm{C}$ and $40{ }^{\circ} \mathrm{C}$, respectively, and $\Delta \mathrm{G}$ values of $-12.4 \mathrm{kcal} \mathrm{mol}^{-1}$ and $-11.1 \mathrm{kcal}$ $\mathrm{mol}^{-1}$, respectively. ${ }^{[16-19]}$

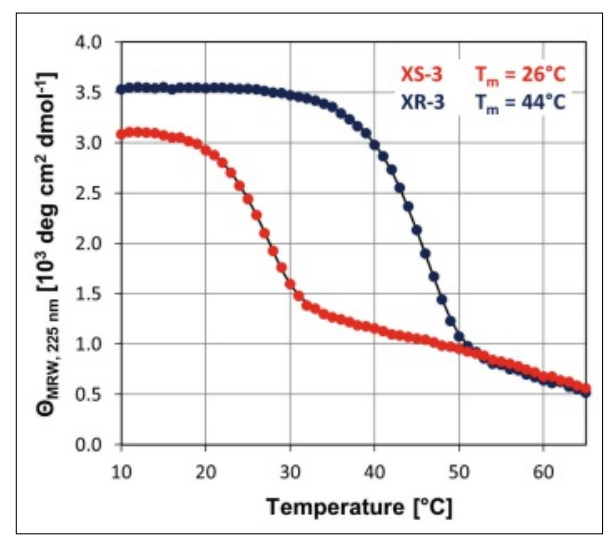

Fig. 5. Thermal denaturation studies exemplified by triple helices derived from the functionalized CMPs XS-3 and XR-3. 
Table 1. Thermodynamic parameters of triple helices derived from the functionalized CMPs Ac[ProHypGly] ${ }_{3}-$ Xaa-Yaa-Gly-[ProHypGly $]_{3} \mathrm{NH}_{2}$ YR2-7, XR2-7, YS2-7, and XS2-7

\begin{tabular}{|c|c|c|c|c|c|}
\hline Entry & CMP & $\begin{array}{l}\text { Xaa } \\
{[\text { [middle }}\end{array}$ & $\begin{array}{l}\text { Yaa } \\
\text { riplet] }\end{array}$ & $\begin{array}{l}\mathrm{T}_{\mathrm{m}}^{\mathrm{a}} \\
{\left[{ }^{\circ} \mathrm{C}\right]}\end{array}$ & $\underset{\left[\mathrm{kcal} \cdot \mathrm{mol}^{-1}\right]}{\Delta \mathrm{bc}^{\mathrm{bc}}}$ \\
\hline \multicolumn{6}{|c|}{ Amidated CMPs } \\
\hline $1^{\mathrm{d}}$ & XR-2 & $(4 R)$ Acp & Pro & 43 & -11.8 \\
\hline $2^{d}$ & XR-3 & $(4 R) \mathrm{Pvp}$ & Pro & 44 & -11.7 \\
\hline $3^{d}$ & YR-2 & Pro & $(4 R)$ Acp & 36 & -10.5 \\
\hline $4^{\mathrm{d}}$ & YR-3 & Pro & $(4 R) \mathrm{Pvp}$ & 32 & -9.2 \\
\hline $5^{e}$ & XS-2 & $(4 S) A c p$ & Pro & 32 & -8.9 \\
\hline $6^{d}$ & XS-3 & $(4 S) \mathrm{Pvp}$ & Pro & 26 & -6.9 \\
\hline $7^{e}$ & YS-2 & Pro & (4S)Acp & 40 & -11.0 \\
\hline $8^{d}$ & YS-3 & Pro & $(4 S) \mathrm{Pvp}$ & 39 & -11.3 \\
\hline \multicolumn{6}{|c|}{ Triazolylated CMPs } \\
\hline $9^{f}$ & XR-4 & $\operatorname{Tzp}\left(\mathrm{CO}_{2} \mathrm{Me}\right)$ & Pro & 39 & $n . d .{ }^{\mathrm{i}}$ \\
\hline $10^{\mathrm{f}}$ & XR-5 & $\operatorname{Tzp}\left(\mathrm{CH}_{2} \mathrm{OH}\right)$ & Pro & 38 & $n . d .^{\mathrm{i}}$ \\
\hline $11^{\mathrm{f}}$ & XR-6 & Tzp (Gal) & Pro & 39 & $n . d .^{\mathrm{i}}$ \\
\hline $12^{\mathrm{f}}$ & XR-7 & Tzp (Glc) & Pro & 39 & $n . d .^{\mathrm{i}}$ \\
\hline $13^{\mathrm{f}}$ & XS-4 & $\operatorname{Tzp}\left(\mathrm{CO}_{2} \mathrm{Me}\right)$ & Pro & 36 & $n . d .{ }^{\mathrm{i}}$ \\
\hline $14^{\mathrm{f}}$ & XS-5 & $\mathrm{Tzp}\left(\mathrm{CH}_{2} \mathrm{OH}\right)$ & Pro & 38 & $n . d .^{\mathrm{i}}$ \\
\hline $15^{\mathrm{f}}$ & XS-6 & $\operatorname{Tzp}(G a l)$ & Pro & 40 & $n . d .^{\mathrm{i}}$ \\
\hline $16^{\mathrm{f}}$ & XS-7 & Tzp $($ Glc $)$ & Pro & 40 & $n . d .^{\mathrm{i}}$ \\
\hline $17^{\mathrm{g}}$ & YR-4 & Pro & $\mathrm{Tzp}\left(\mathrm{CO}_{2} \mathrm{Me}\right)$ & 36 & $n . d .{ }^{\mathrm{i}}$ \\
\hline $18^{\mathrm{g}}$ & YR-5 & Pro & $\mathrm{Tzp}\left(\mathrm{CH}_{2} \mathrm{OH}\right)$ & 35 & $n . d .^{\mathrm{i}}$ \\
\hline $19^{\mathrm{g}}$ & YR-6 & Pro & Tzp(Gal) & 37 & $n . d .{ }^{\mathrm{i}}$ \\
\hline $20^{\mathrm{g}}$ & YR-7 & Pro & Tzp(Glc) & 37 & $n . d .^{\mathrm{i}}$ \\
\hline $21^{\mathrm{h}}$ & YS-4 & Pro & $\mathrm{Tzp}\left(\mathrm{CO}_{2} \mathrm{Me}\right)$ & 35 & $n . d .^{\mathrm{i}}$ \\
\hline $22^{\mathrm{h}}$ & YS-5 & Pro & $\mathrm{Tzp}\left(\mathrm{CH}_{2} \mathrm{OH}\right)$ & 36 & $n . d .^{\mathrm{i}}$ \\
\hline $23^{\mathrm{h}}$ & YS-6 & Pro & Tzp(Gal) & 37 & $n . d .^{\mathrm{i}}$ \\
\hline $24^{\mathrm{h}}$ & YS-7 & Pro & $\operatorname{Tzp}($ Glc $)$ & 37 & $n . d .{ }^{\mathrm{i}}$ \\
\hline \multicolumn{6}{|c|}{ Reference CMPs } \\
\hline $25^{\mathrm{e}}$ & 8 & Pro & (4R)Нур & 43 & -12.4 \\
\hline $26^{e}$ & 9 & Pro & Pro & 40 & -11.1 \\
\hline
\end{tabular}

${ }^{\mathrm{a}} \mathrm{T}$ at a heating rate of $1^{\circ} \mathrm{C} / 100 \mathrm{~s}\left( \pm 1^{\circ} \mathrm{C}\right) .{ }^{\mathrm{b}} \mathrm{Data}$ at a heating rate of $0.1^{\circ} \mathrm{C} / 72 \mathrm{~s} .{ }^{\circ} \Delta \mathrm{G}$ at $25^{\circ} \mathrm{C}$ $\left( \pm 0.2 \mathrm{kcal} / \mathrm{mol}^{-1}\right) .{ }^{\mathrm{d}}$ Data taken from ref [19]. ${ }^{e}$ Data taken from ref [18]. ${ }^{\text {fData }}$ taken from ref [17]. 9Data taken from ref [15]. 'Data taken from ref [16]. 'not determined.

Comparable thermal stabilities were observed with the functionalized collagen triple helices derived from the CMPs XR-2 and XR-3 bearing ( $4 R$ )-configured Acp or Pvp residues in the Xaa position indicating that these residues do not disturb the supramolecular triple helical fold (Table 1, entries 1 and 2). ${ }^{[19]}$ In contrast, incorporation of $(4 S)$-configured Acp or Pvp residues in the Xaa position (CMPs XS-2 and XS-3) caused the biggest destabilization of the triple helix within the examined series (Table 1, entries 5 and 6). ${ }^{[19]}$ This finding can be easily understood by considering the steric environment and the interstrand $\mathrm{H}$-bonds of residues in the Xaa position: Within (4S)-amidoproline residues the transannular $\mathrm{H}$-bond competes with the interstrand $\mathrm{H}$-bond that holds the three collagen single strands together. ${ }^{[18]}$ In addition, the substituent installed at the $(4 S)$ configured $\gamma$-carbon points to the inside of the triple helix and clashes sterically with the residue in the Yaa position of the neighboring strand ${ }^{[19]}$ In contrast, diastereomeric $(4 R)$-configured amidoprolines neither interfere with the interstrand $\mathrm{H}$-bonding nor do they cause steric repulsions with other residues since the substituent points to the outside of the triple helix. ${ }^{[18,19]}$ Even sterically demanding residues such as tertbutyl groups are readily tolerated when installed at $(4 R)$-configured residues in the Xaa position.
In the Yaa position the situation is different since the amino acids in this position are not involved in interstrand $\mathrm{H}$-bonding. Here substituents that are installed at $(4 R)$ configured amidoprolines (CMPs YR-2 and YR-3) interfere sterically with residues from neighboring strands whereas substituents at (4S)-configured amidoprolines (CMPs YS-2 and YS-3) point to the outside of the triple helix and are readily tolerated (Table 1, entries 3, 4, 7 and 8). ${ }^{[19]}$

Within the most stable functionalized collagen triple helices derived from the functionalized CMPs XR-2 and XR-3 as well as YS-2 and YS-3 the preferred ring puckering of the functionalized residues differ from the ring puckering observed in crystal structures for Pro and Hyp residues in natural collagen: Whereas Pro residues in the Xaa position occur predominantly with $\mathrm{C}(4)$-endo puckers in natural collagen $(4 R)$-configured amidoproline residues adopt preferentially $\mathrm{C}(4)$-exo ring puckers. Likewise, the $\mathrm{C}(4)$-endo puckering of $(4 S)$ configured amidoproline residues does not match the $\mathrm{C}(4)$-exo puckering of Hyp residues in the Yaa position of natural collagen. Thus, the studies also showed that the ring puckering of the proline residues is not as critical for the supramolecular assembly as steric constraints or interference with the interstrand H-bonds. ${ }^{[19]}$

The thermal stability of the collagen triple helices containing the triazolyl proline residues in either the Xaa or Yaa position were rather similar, which was expected based on the conformational properties of the Ac-Tzp-OMe amino acids. ${ }^{[15-17]}$ All of the Tzp-containing CMPs formed triple helices with $\mathrm{T}_{\mathrm{m}}$ values in a range of $35-40{ }^{\circ} \mathrm{C}$. Within this series the same trends with respect to steric effects were observed as for the acylated CMPs.

\section{Conclusions}

In conclusion, azidoproline-containing CMPs are versatile tools for installing functional moieties in collagen triple helices by either 'click chemistry' or Staudinger reduction followed by acylation. Moreover, the presented functionalized CMPs provided insight into the factors that are critical for the supramolecular assembly of the collagen triple helix: a) Sterically demanding moieties disturb the supramolecular assembly the least when they are installed at $(4 S)$-configured residues in the Yaa position or $(4 R)$-configured residues in the Xaa position since the bulky moieties point in both cases to the outside of the collagen triple helix, b) residues bearing $\mathrm{H}$-bond donors that interfere with the interstrand $\mathrm{H}$-bonds within collagen weaken the triple helix significantly, and c) the ring puckering is less important compared to steric and 
unfavorable H-bonding effects. Thus, our research provided a detailed guide for the functionalization of collagen without or by deliberately disturbing the supramolecular assembly. We are currently using these insights for the development of cross-linked collagen based materials bearing moieties that are envisioned to be useful for applications in e.g. wound healing, bone formation and tissue engineering.

Received: September 18, 2013

[1] For reviews see: a) G. B. Fields, D. J. Prockop, Biopolymers 1996, 40, 345; b) J. Engel, H. P. Bachinger Top. Curr. Chem. 2005, 247, 7; c) B. Brodsky, G. Thiagarajan, B. Madhan, K. Kar, Biopolymers 2008, 89, 345; d) M. D. Shoulders, R. T. Raines, Annu. Rev. Biochem. 2009, 78, 929.

[2] For reviews see: a) G. B. Fields, Org. Biomol. Chem. 2010, 8, 1237; b) D. E. Przybyla, J. Chmielewski, Biochemistry 2010, 49, 4411; c) S. M. Yu, Y. Li, D. Kim, Soft Matter 2011, 7, 7927.

[3] G. Fischer, Chem. Soc. Rev. 2000, 29, 119.

[4] a) J. Bella, M. Eaton, B. Brodsky, H. M. Berman, Science 1994, 266, 75; b) K. Okuyama, C Hongo, R. Fukushima, G. G. Wu, H. Narita, K. Noguchi, Y. Tanaka, N. Nishino, Biopolymers 2004, 76, 367.

[5] a) P. Krattiger, R. Kovàsy, J. D. Revell, S. Ivan, H. Wennemers, Org. Lett. 2005, 7, 1101; b) P. Krattiger, R. Kovàsy, J. D. Revell, H Wennemers, QSAR Comb. Sci. 2005, 24, 1158; c) J. D. Revell, D. Gantenbein, P. Krattiger, H. Wennemers, Biopolymers (Pept. Sci.) 2006, 84,105 ; d) J. Grun, J. D. Revell, M. Conza, H. Wennemers, Bioorg. Med. Chem. 2006, 14, 6197; e) J. D. Revell, H. Wennemers, Tetrahedron 2007, 63, 8420; f) J. D. Revell,
H. Wennemers, Adv. Synth. Catal. 2008, 350, 1046; g) M. Messerer, H. Wennemers, Synlett 2011, 499 .

[6] a) M. Wiesner, J. D. Revell, H. Wennemers, Angew. Chem. Int. Ed. 2008, 47, 1871; b) M. Wiesner, M. Neuburger, H. Wennemers, Chem. Eur. J. 2009, 15, 10103; c) M. Wiesner, G. Upert, G. Angelici, H. Wennemers, J. Am. Chem. Soc. 2010, 132, 6; d) M. Wiesner, H. Wennemers, Synthesis 2010, 1568; e) Y. Arakawa, M Wiesner, H. Wennemers, Adv. Synth. Catal. 2011, 353, 1201; f) Y. Arakawa, H. Wennemers, ChemSusChem 2013, 6, 242; g) M. Wiesner, J. D. Revell, S. Tonazzi, H. Wennemers, J. Am Chem. Soc. 2008, 130, 5610; h) J. Duschmalé, H. Wennemers, Chem. Eur. J. 2012, 18, 1111 i) J. Duschmalé, J. Wiest, M. Wiesner, H Wennemers, Chem. Sci. 2013, 4, 1312; j) R. Kastl, H. Wennemers, Angew. Chem. Int. Ed 2013, 52, 7228; k) F. Bächle, J. Duschmalé, C. Ebner, A. Pfaltz, H. Wennemers, Angew. Chem. Int. Ed. 2013, 52, 12619.

[7] a) H. Wennemers, M. Conza, M. Nold, P. Krattiger, Chem. Eur. J. 2001, 7, 3342; b) M. Conza, H. Wennemers, J. Org. Chem. 2002, 67, 2696; c) H. Wennemers, M. Nold, M. Conza, K. J. Kulicke, M. Neuburger, Chem Eur. J. 2003, 9, 442; d) P. Krattiger, H. Wennemers, Synlett 2005, 4, 706; e) J. Bernard, H. Wennemers, Org. Lett. 2007, 9, 4283; f) C. F. J. Faul, P. Krattiger, B. Smarsly, H. Wennemers, J. Mater. Chem. 2008, 18, 2962.

[8] a) M. Kümin, L.-S. Sonntag, H. Wennemers, J. Am. Chem. Soc. 2007, 129, 466; b) R. S Erdmann, M. Kuemin, H. Wennemers, Chimia, 2009, 63, 197; c) R. A. Kramer, K. H. Bleicher, H. Wennemers, Helv. Chim. Acta 2012, 95, 2621.

[9] a) G. Upert, F. Bouillère, H. Wennemers, Angew. Chem. Int. Ed. 2012, 51, 4231; b) C. Kroll, R. Mansi, F. Braun, S. Dobitz, H. Maecke, H. Wennemers, J. Am. Chem. Soc. 2013, 135, 16793.

[10] a) M. Kuemin, S. Schweizer, C. Ochsenfeld,
H. Wennemers, J. Am. Chem. Soc. 2009, 131, 15474 ; b) M. Kuemin, J. Engel, H. Wennemers, J. Pept. Sci. 2010, 16, 596.

[11] L.-S. Sonntag, S. Schweizer, C. Ochsenfeld, H. Wennemers, H. J. Am. Chem. Soc. 2006, 128, 14697.

[12] M. Kuemin, Y. A. Nagel, S. Schweizer, F. W. Monnard, C. Ochsenfeld, H. Wennemers, Angew. Chem. Int. Ed. 2010, 49, 6324.

[13] For examples, see: a) S. K. Holmgren, K. M. Taylor, L. E. Bretscher, R. T. Raines, Nature 1998, 392, 666; b) S. K. Holmgren, L. E. Bretscher, K. M. Taylor, R. T. Raines, Chem. Biol. 1999, 6, 63; c) L. E. Bretscher, C. L. Jenkins, K. M. Taylor, M. L. DeRider, R. T. Raines, J. Am. Chem. Soc. 2001, 123, 777; d) J. A. Hodges, R. T. Raines, J. Am. Chem. Soc. 2003, 125, 9262; e) M. D. Shoulders, J. A. Hodges, R. T. Raines, J. Am. Chem. Soc. 2006, 128,8112 ; f) M. D. Shoulders, K. A. Satyshur, K. T. Forest, R. T. Raines, Proc. Natl. Acad. Sci. USA 2010, 107, 559; g) S. A. Cadamuro, R. Reichold, U. Kusebauch, H.-J. Musiol, C. Renner, P. Tavan, L. Moroder, Angew. Chem., Int. Ed. 2008, 47, 2143 .

[14] R. S. Erdmann, H. Wennemers, Synthesis 2009, 143.

[15] R. S. Erdmann, H. Wennemers, J. Am. Chem. Soc. 2010, 132, 13957.

[16] R. S. Erdmann, H. Wennemers, Org. Biomol. Chem. 2012, 10, 1982.

[17] R. S. Erdmann, H. Wennemers, Bioorg. Med. Chem. 2013, 21, 3565.

[18] R. S. Erdmann, H. Wennemers, Angew. Chem., Int. Ed. 2011, 50, 6835 .

[19] R. S. Erdmann, H. Wennemers, J. Am. Chem. Soc. 2012, 134, 17117.

[20] a) M. P. Hinderaker, R. T. Raines, Protein Sci. 2003, 12, 1188; b) A. Choudhary, D. Gandla, G. R. Krow, R. T. Raines, J. Am. Chem. Soc. 2009 , 131, 7244; c) R. W. Newberry, B. VanVeller, R. T. Raines J. Am. Chem. Soc. 2013, 135, 7843.

[21] K. Mizuno, S. P. Boudko, J. Engel, H. P. Bachinger, Biophys. J. 2010, 98, 3004. 